\title{
Gold Reflectors in Infra-Red Heating
}

\author{
J. L. Lambert \\ Heraeus Quarzschmelze, Hanau, West Germany
}

Heating by means of medium wave or short wave infra-red radiation is an essential feature of a wide variety of industrial processes. Its applications are to be found, for example, in the textile and papermaking industries, in printing, in the manufacture of plastics and of laminated glass, and in cooking and the drying of foodstuffs. Generally it is used with most effect in conveyor type ovens where continuous processing is involved and where poorly conducting materials are to be heated rapidly.

The high temperature source employed is either a tungsten filament operating at around $2200^{\circ} \mathrm{C}$-for short wave-or a nickel-chromium spiral filament running at $800^{\circ} \mathrm{C}$ or over for medium wave heating. In either case the filament is enclosed in a tubular envelope of quartz glass, a material which will withstand the high temperature and which is also transparent to infra-red. The envelope thus remains relatively cool and so minimises the loss of radiant heat to the surrounding atmosphere.
This type of construction provides a simple and compact unit giving rapid heating and cooling times with ease of maintenance.

In many cases these infra-red units are located above the material to be heated, and an efficient means of reflecting the heat must be employed. While external reflectors can be arranged, an improved technique recently developed involves applying a conventional gold coating to the reverse side of the quartz glass envelope. This gives a highly efficient reflector for infra-red, more than 99 per cent, particularly when employed in the form of twin tubes. Improved performance and of course the permanent maintenance of the high reflecting gold surface are ensured since it is not directly exposed to possibly unclean environments.

For a more detailed account of the fundamentals of infra-red heating and of the types of equipment available an earlier paper by the writer may be found useful (Electrical Review, 1974, 194, (22), 681-684).

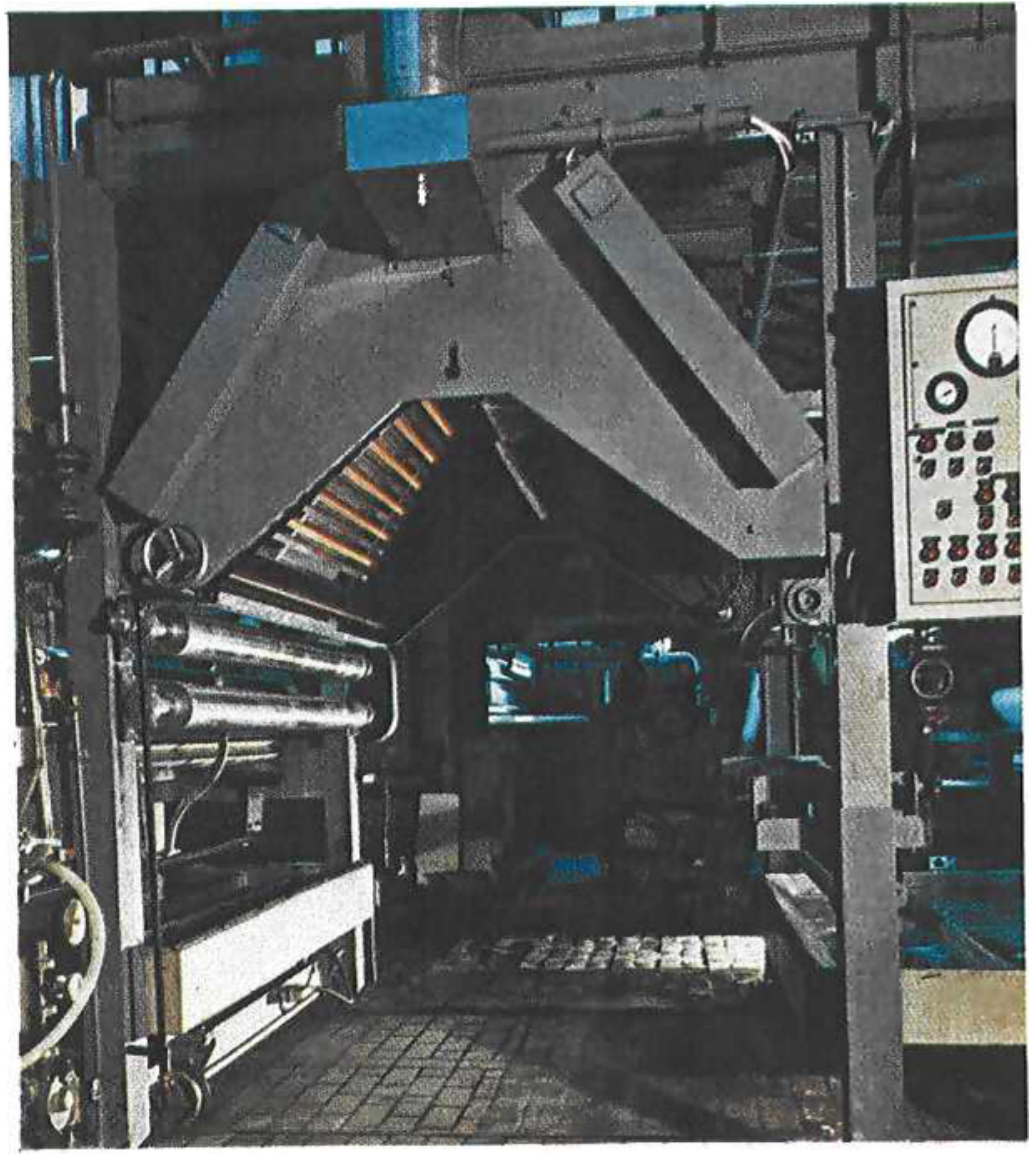

The maintenence of a high efficiency in the infra-red heating section of a paper converting machine is achieved by the use of gold-coated twin-tube quartz glass radiators 